OPEN ACCESS

Edited by: Jumpei Sasabe,

Keio University, Japan

Reviewed by:

Kouji Uda,

Kōchi University, Japan

Volker F. Wendisch,

Bielefeld University, Germany

*Correspondence:

Tadao Oikawa

oikawa@kansai-u.ac.jp

${ }^{\dagger}$ Present address:

Shiro Kato,

International Institute of Rare Sugar Research and Education, Kagawa

University, Takamatsu, Japan

Specialty section:

This article was submitted to Microbial Physiology and Metabolism,

a section of the journa

Frontiers in Microbiology

Received: 12 December 2017

Accepted: 21 February 2018

Published: 07 March 2018

Citation:

Kato S and Oikawa T (2018) A Novel Bifunctional Amino Acid Racemase With Multiple Substrate Specificity, MalY From Lactobacillus sakei LT-13:

Genome-Based Identification and Enzymological Characterization.

Front. Microbiol. 9:403.

doi: 10.3389/fmicb.2018.00403

\section{A Novel Bifunctional Amino Acid Racemase With Multiple Substrate Specificity, MalY From Lactobacillus sakei LT-13: Genome-Based Identification and Enzymological Characterization}

\author{
Shiro Kato ${ }^{1+}$ and Tadao Oikawa ${ }^{1,2 *}$ \\ ${ }^{1}$ High Technology Research Core, Kansai University, Suita, Japan, ${ }^{2}$ Department of Life Science and Biotechnology, Faculty \\ of Chemistry, Materials, and Bioengineering, Kansai University, Suita, Japan
}

The Lactobacillus sakei strain LK-145 isolated from Moto, a starter of sake, produces potentially large amounts of three D-amino acids, D-Ala, D-Glu, and D-Asp, in a medium containing amylase-digested rice as a carbon source. The comparison of metabolic pathways deduced from the complete genome sequence of strain LK-145 to the type culture strain of Lactobacillus sakei strain LT-13 showed that the L- and D-amino acid metabolic pathways are similar between the two strains. However, a marked difference was observed in the putative cysteine/methionine metabolic pathways of strain LK145 and LT-13. The cystathionine $\beta$-lyase homolog gene malY was annotated only in the genome of strain LT-13. Cystathionine $\beta$-lyase is an important enzyme in the cysteine/methionine metabolic pathway that catalyzes the conversion of L-cystathionine into L-homocysteine. In addition to malY, most genome-sequenced strains of $L$. sakei including LT-13 lacked the homologous genes encoding other putative enzymes in this pathway. Accordingly, the cysteine/methionine metabolic pathway likely does not function well in almost all strains of $L$. sakei. We succeeded in cloning and expressing the malY gene from strain LT-13 (Ls-ma/Y) in the cells of Escherichia coli BL21 (DE3) and characterized the enzymological properties of $L s$-MalY. Spectral analysis of purified Ls-MalY showed that Ls-MalY contained a pyridoxal 5'-phosphate (PLP) as a cofactor, and this observation agreed well with the prediction based on its primary structure. $L s$ MalY showed amino acid racemase activity and cystathionine $\beta$-lyase activity. Ls-MalY showed amino acid racemase activities in various amino acids, such as Ala, Arg, Asn, Glu, Gln, His, Leu, Lys, Met, Ser, Thr, Trp, and Val. Mutational analysis revealed that the $\varepsilon$-amino group of Lys233 in the primary structure of Ls-MalY likely bound to PLP, and Lys233 was an essential residue for Ls-MalY to catalyze both the amino acid racemase and $\beta$-lyase reactions. In addition, Tyr123 was a catalytic residue in the amino 
acid racemase reaction but strongly affected $\beta$-lyase activity. These results showed that Ls-MalY is a novel bifunctional amino acid racemase with multiple substrate specificity; both the amino acid racemase and $\beta$-lyase reactions of $L s$-MalY were catalyzed at the same active site.

Keywords: D-amino acid, amino acid racemase, Lactobacillus sakei, genome analysis, lactic acid bacteria, maltose regulon, cystathionine $\beta$-lyase, bifunctional enzyme

\section{INTRODUCTION}

A main source of D-amino acids in bacteria is amino acid racemase, which catalyzes the interconversion of $\mathrm{D}$ - and L-enantiomers of amino acids. In general, amino acid racemase is classified into two groups: pyridoxal $5^{\prime}$-phosphate (PLP)independent enzyme and PLP-dependent enzyme. The PLPindependent amino acid racemase includes glutamate racemase (Choi et al., 1992; Yoshimura et al., 1993), aspartate racemase (Fujii et al., 2015), and proline racemase (Cardinale and Abeles, 1968) and contains two Cys residues as a catalytic residue (Choi et al., 1992; Washio et al., 2016). In contrast, PLPdependent amino acid racemases such as alanine racemase (Oikawa et al., 2006) and arginine racemase (Matsui et al., 2009) requires PLP as a cofactor. Although amino acid racemases from various bacteria have been studied extensively, novel amino acid racemases may still be discovered. $\mathrm{Li}$ and $\mathrm{Lu}$ demonstrated that D-Arg was metabolized after racemization into L-Arg using a novel amino acid racemase consisting of two PLP-independent dehydrogenases in Pseudomonas aeruginosa (Li and Lu, 2009). A protein annotated as a $\gamma$-aminobutyrate aminotransferase from Lactobacillus otakiensis or Lactobacillus buchneri has been identified as a novel PLP-dependent epimerase that converts L-Ile into D-allo-Ile (Mutaguchi et al., 2013b). Recently, the RacX from Bacillus subtilis and YgeA from Escherichia coli MG1655 have been shown to be a novel amino acid racemase with broad substrate specificity (Miyamoto et al., 2017). The physiological roles of $\mathrm{D}$-amino acid in bacteria have long been considered, but only as providing an essential component in bacterial peptidoglycan and antibiotics. However, recent studies of bacterial D-amino acids have revealed that some D-amino acids relate to other physiological roles, such as the remodeling of bacterial peptidoglycan in the stationary phase (Lam et al., 2009) and the dispersion of bacterial biofilm (Kolodkin-Gal et al., 2010). These attractive studies in the research field of D-amino acids motivate researchers such as ourselves to find a novel amino acid racemase and a novel role for D-amino acid in bacteria.

Lactic acid bacteria are Gram-positive lactic acid-producing bacteria and are used as starters in fermented foods such as Japanese sake, wine, vinegar, yogurt, and cheese. Several research groups, including our lab group, have clarified that fermented foods contain significant amount of D-amino acids, and such D-amino acids are mainly produced by lactic acid bacteria (Gogami et al., 2011; Kato et al., 2011; Mutaguchi et al., 2013a). Our group reported for the first time that D-Ala, D-Asp, and D-Glu in Japanese sake increase the taste and total balance of the taste of sake, and other D-amino acids showed no effect
(Okada et al., 2013). Recently, we analyzed and reported the complete genome sequences of two Lactobacillus sakei strains, LK-145 (Kato and Oikawa, 2017a) and LT-13 (Kato and Oikawa, 2017b). Strain LK-145 was isolated from a Japanese sake seller as a high D-amino acid producer (Gogami et al., 2011) and strain LT-13 was isolated from Moto, a starter of sake, as a low Damino acid producer, using a medium of amylase digested rice as a carbon source. The overall genome structure of strain LK-145 was similar to that of strain LT-13 (Kato and Oikawa, 2017a,b) or L. sakei strain 23K (Chaillou et al., 2005), the first genome sequenced strain of L. sakei. However, a marked difference was observed in the putative cysteine/methionine metabolic pathways of strain LK-145 and LT-13. The cystathionine $\beta$-lyase homolog gene (accession no. BAX66038), malY was only annotated in the genome of strain LT-13. Accordingly, the gene product of malY is expected to be involved in the differences in D-amino acid productivity between strain LK-145 and strain LT-13.

In this study, we tried to clone and express the malY gene from the genome of strain LT-13 (Ls-malY) in the cells of E. coli BL21 (DE3) and to characterize the enzyme properties of $L s$-MalY in vitro to elucidate the relationship between Ls-MalY and the D-amino acid metabolism of strain LT-13.

\section{MATERIALS AND METHODS}

\section{Reagents}

Amino acids and pyruvic acid were purchased from Wako Pure Chemicals, Co., Ltd. (Japan), Watanabe Chemical Industries, Ltd. (Japan) or Sigma Japan. Restriction enzymes were from New England Biolabs Japan. KOD -plus ver. 2 DNA polymerase was from Toyobo, Co., Ltd. (Japan). Methanol and acetonitrile were from Kanto Kagaku, Co., Ltd. (Japan). Molecular weight standards for gel filtration chromatography were from GE Healthcare Japan. All other reagents were of analytical or molecular biology grade.

\section{Cloning and Expression of MalY Gene from Genome of $L$. sakei Strain LT-13 into Cells of E. coli BL21 (DE3)}

The Ls-malY gene was amplified by polymerase chain reaction (denaturing, $10 \mathrm{~s}$ at $98^{\circ} \mathrm{C}$; annealing, $30 \mathrm{~s}$ at $58^{\circ} \mathrm{C}$; elongation, $1 \mathrm{~min} 30 \mathrm{~s}$ at $68^{\circ} \mathrm{C} ; 30$ cycles) using L. sakei LT- 13 chromosomal DNA as a template with the primers MalY F and MalY R (Table 1). Since the amplified DNA fragment contained two NdeI sites at $5^{\prime}$ terminus and in the coding region of $L s$-malY gene, the fragment was digested with XhoI and partially with NdeI and 
then was subjected to agarose gel electrophoresis. The desired DNA fragment of approximately $1.2 \mathrm{~kb}$ was extracted from the gel, purified, and ligated into a pET-22b $(+)$, yielding pE-MalY. E. coli BL21(DE3) cells harboring pE-MalY was cultivated in auto-induction medium (Grabski et al., 2005; Studier, 2005) containing ampicillin $(100 \mu \mathrm{g} / \mathrm{mL})$. After cultivation at $30^{\circ} \mathrm{C}$ for $24 \mathrm{~h}$, cells were harvested by centrifugation at $10,000 \times g$ at $4^{\circ} \mathrm{C}$ for $5 \mathrm{~min}$.

\section{Site-Directed Mutagenesis}

Two plasmids for expression of Ls-MalY single point mutants, pE-MalY Y123A and pE-MalY K233A, were prepared from $\mathrm{pE}$ MalY using a quick-change mutagenesis method with primers listed in Table 1. The presence of the mutation and fidelity of the mutagenesis was confirmed by sequencing. The mutated malY genes were expressed as described for the wild-type (WT) gene.

\section{Purification of Ls-MalY and Mutants}

The harvested transformant cells were resuspended in a $20 \mathrm{mM}$ potassium phosphate buffer ( $\mathrm{pH} 7.4$ ) containing $0.5 \mathrm{M} \mathrm{KCl}$ and $20 \mathrm{mM}$ imidazole (Buffer A) and disrupted by ultrasonication and centrifuged to remove cell debris. The supernatant was applied to a column of Ni Sepharose ${ }^{\mathrm{TM}} 6$ Fast Flow resin $(4 \mathrm{~mL}$ bed volume, GE Healthcare Japan) that previously equilibrated with Buffer A. After the column was washed with Buffer A, the enzyme was eluted with $20 \mathrm{mM}$ potassium phosphate buffer $(\mathrm{pH} 7.4)$ containing $0.5 \mathrm{M} \mathrm{KCl}$ and $0.2 \mathrm{M}$ imidazole. The purified enzyme was dialyzed against $20 \mathrm{mM}$ potassium phosphate buffer $(\mathrm{pH} 7.5)$ and stored at $-80^{\circ} \mathrm{C}$ until use.

\section{Gel Filtration Chromatography}

The molecular weight of Ls-MalY was identified by sizeexclusion chromatography using an ÄKTA purifier system (GE Healthcare Japan) with a Superdex 200 Increase 10/300 GL column (GE Healthcare Japan). Potassium phosphate buffer (20 mM, pH 7.5) containing 0.15 M KCl (Buffer B) was used as the isocratic mobile phase, and the flow rate was $0.75 \mathrm{~mL} / \mathrm{min}$. Thyroglobulin, apoferritin, $\beta$-amylase, bovine serum albumin (BSA), and carbonic anhydrase (150-200 $\mu \mathrm{g} /$ protein) were dissolved in Buffer B and used as a molecular weight marker.

\section{Enzyme Assay}

The standard assay conditions for analysis of $L s$-MalY racemase activity were as follows: reaction mixture $(1 \mathrm{~mL})$ containing a

TABLE 1 | Primers used in this study.

\begin{tabular}{|c|c|}
\hline Primer & Sequence $\left(5^{\prime}\right.$ to $\left.3^{\prime}\right)$ \\
\hline MalY F & TTCGATAGCATATG ${ }^{a}$ ACGAAGTITGAC ПTG \\
\hline MalY R & TाTCAGCTCGAG ${ }^{\mathrm{b}}$ TCTCTGCTGGATAGCTTC \\
\hline Y123A F & TAGTCCTTGTGCGGATGCGTTATTAATAC \\
\hline Y123AR & AAACGCATCCGCACAAGGACTAAAAGTAAC \\
\hline K233A F & TCTGCCAGCGCGTCATTTAATATCCCAGC \\
\hline K233A R & ATATTAAATGACGCGCTGGCAGACGTTATC \\
\hline
\end{tabular}

$\mathrm{Ndel}^{\mathrm{a}}$ and $\mathrm{Xhol} \mathrm{l}^{\mathrm{b}}$ sites are underlined.
$50 \mathrm{mM}$ potassium phosphate ( $\mathrm{pH} 7.5$ ), $50 \mathrm{mM}$ substrate, $50 \mu \mathrm{M}$ PLP and $L s$-MalY $(200 \mu \mathrm{g})$, incubated at $30^{\circ} \mathrm{C}$ for $60 \mathrm{~min}$. After stopping the reaction by boiling, the supernatant was subjected to high-performance liquid chromatography (HPLC) analysis, which was performed as described previously (Gogami et al., 2011; Kato et al., 2015; Washio et al., 2016).

The $\beta$-lyase activity of $L s$-MalY was assayed by quantifying $\alpha$-keto acid using the 3-methyl-2-benzothiazolone hydrazone (MBTH) method (Soda, 1968). The standard assay conditions were as follows: after incubation at $30^{\circ} \mathrm{C}$ for $60 \mathrm{~min}$, the reaction mixture $(1 \mathrm{~mL})$ consisted of a $50 \mathrm{mM}$ potassium phosphate ( $\mathrm{pH}$ 7.5), $50 \mathrm{mM}$ substrate, and $50 \mu \mathrm{M}$ PLP and Ls-MalY $(200 \mu \mathrm{g})$ was mixed with $100 \mu \mathrm{L}$ of $25 \%(\mathrm{w} / \mathrm{v})$ trichloroacetic acid to stop the reaction. Sodium acetate buffer (1 M; pH $5.0 ; 1900 \mu \mathrm{L}$ ) and $800 \mu \mathrm{L}$ of $0.1 \%(\mathrm{w} / \mathrm{v}) \mathrm{MBTH}$ were added to the mixture, and the mixture was incubated at $50^{\circ} \mathrm{C}$ for $30 \mathrm{~min}$. After further incubation at room temperature for $20 \mathrm{~min}$, the absorbance of the mixture was measured at $316 \mathrm{~nm}$.

\section{pH-Activity and Temperature-Activity Profiles}

The effects of $\mathrm{pH}$ and temperature on racemase or $\beta$-lyase activity were examined using L-Ala or L-Cys as a substrate, respectively. The optimum $\mathrm{pH}$ for enzyme activity was determined by assaying the enzyme at $30^{\circ} \mathrm{C}$ at $\mathrm{pH} 4.0$ to $12.0[\mathrm{pH} 4.0-12.0(50 \mathrm{mM}$ Britton-Robinson); $\mathrm{pH}$ 4.0-6.0 (50 mM acetate); $\mathrm{pH}$ 6.0-8.0 (50 mM potassium phosphate), $\mathrm{pH} 9.5-10.0$ (50 mM borate), and $\mathrm{pH}$ 10.0-11.0 (50 mM carbonate)]. The optimum temperature was analyzed at $\mathrm{pH} 7.5$ using $50 \mathrm{mM}$ potassium phosphate from 20 to $55^{\circ} \mathrm{C}$.

\section{Kinetic Analysis}

The $L$ s-MalY enzyme reaction was performed at $40^{\circ} \mathrm{C}$ (racemase reaction) or $35^{\circ} \mathrm{C}$ ( $\beta$-lyase reaction) with a reaction mixture consisting of $50 \mathrm{mM}$ borate buffer ( $\mathrm{pH} 10.0$ ), $50 \mu \mathrm{M}$ PLP, Ls-MalY (200 $\mu \mathrm{g})$, and substrate. The reaction time was 10$120 \mathrm{~min}$ and the substrate concentrations were $0.5,1,2,5,10,20$, or $50 \mathrm{mM}$ for the racemase reaction and $1,2,3,4,5$, or $7.5 \mathrm{mM}$ for the $\beta$-lyase reaction. Kinetic parameters for each reaction were determined using a Lineweaver-Burk plot (Lineweaver and Burk, 1934).

\section{Structural Modeling}

A structural model of $L s$-MalY was created with modeler software ver. 9.15 using the E. coli MalY (Ec-MalY) structure (PDB code, $1 \mathrm{D} 2 \mathrm{~F})$ as a template.

\section{Spectral Analysis}

Spectral changes during the $\beta$-lyase reaction of WT Ls-MalY with $\mathrm{L}$ - or D-Cys were analyzed by measuring the UV-vis absorption spectrum of the reaction mixture incubated at $30^{\circ} \mathrm{C}$, which consists of $50 \mathrm{mM}$ potassium phosphate ( $\mathrm{pH}$ 7.5), Ls-MalY ( $2 \mathrm{mg} / \mathrm{mL}$ ), and substrate. Dose dependence was assessed by measuring the spectrum after incubation for $30 \mathrm{~min}$ at a substrate concentration range of 0 to $10 \mathrm{mM}$. Reaction time-dependent 
changes in the spectrum of $L s$-MalY with $10 \mathrm{mM}$ substrate were monitored from 0 to $60 \mathrm{~min}$.

\section{RESULTS}

\section{Identification of a Candidate Gene Related to D-Amino Acid Metabolism}

From the comparison of putative metabolic pathways constructed using the KEGG automatic annotation server (Moriya et al., 2007), no difference was observed in the D-amino acid metabolic pathway of L. sakei strains LT-13 and LK-145. However, a remarkable difference was observed in the putative Cys/Met metabolic pathway: a putative cystathionine $\beta$-lyase was identified only in the expected pathway of strain LT-13. The strain LT-13 genome contains an approximately $8-\mathrm{kb}$ insertion region, including a putative cystathionine $\beta$-lyase gene malY (LACBS_00576), compared to strain LK-145 (Figure 1). Cystathionine $\beta$-lyase (EC 4.4.1.8) catalyzes a reaction that degrades L-cystathionine into L-homocysteine, ammonia and pyruvate, but no putative pathway for the biosynthesis of L-cystathionine and degradation/utilization of L-homocysteine was conserved in the strain LT-13 pathway. Overexpression of the cystathionine $\beta$-lyase gene (malY) from E. coli partially compensates the growth defect of the D-Ala-auxotrophic strain of E. coli (Kang et al., 2011), but the details remain unknown. Therefore, to examine the enzyme function of $L s$-MalY, the $L s-m a l Y$ gene was cloned and overexpressed in E. coli.

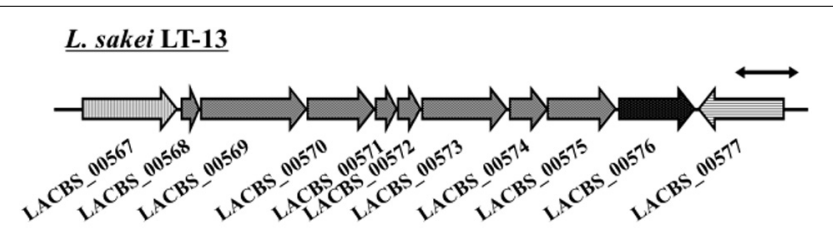

\section{L. sakei LK-145}

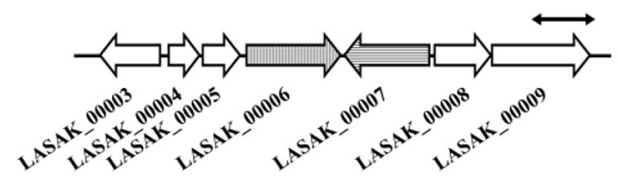

FIGURE 1 | Genome map near malY gene of Lactobacillus sakei LT-13. A portion of $L$. sakei LT-13 genome near malY gene was compared to counter-region of $L$. sakei LK-145 genome. Solid bar with double arrowheads indicates $1 \mathrm{~kb}$ length. LACBS_00567: drug: $\mathrm{H}(+)$ antiporter; LACBS_00568: PRD domain protein; LACBS_00569: PRD domain protein; LACBS_00570: aminopeptidase; LACBS_00571: PTS system lactose/cellobiose-specific iia; LACBS_00572: PTS system lactose/cellobiose-specific iib; LACBS_00573: PTS system lactose/cellobiose-specific iic; LACBS_00574: RDD family protein; LACBS_00575: aminopeptidase; LACBS_00576: maltose regulon modulator; LACBS_00577: glutathione reductase; LASAK_00003: zinc-containing alcohol dehydrogenase; LASAK_00004: MarR family transcriptional regulator; LASAK_00005: hypothetical protein; LASAK_00006: drug:H(+) antiporter; LASAK_00007: glutathione reductase; LASAK_00008: glycine/betaine/carnitine/choline ABC transporter ATP-binding subunit; and LASAK_00009: glycine/betaine/carnitine/choline ABC transporter substrate binding protein/permease.
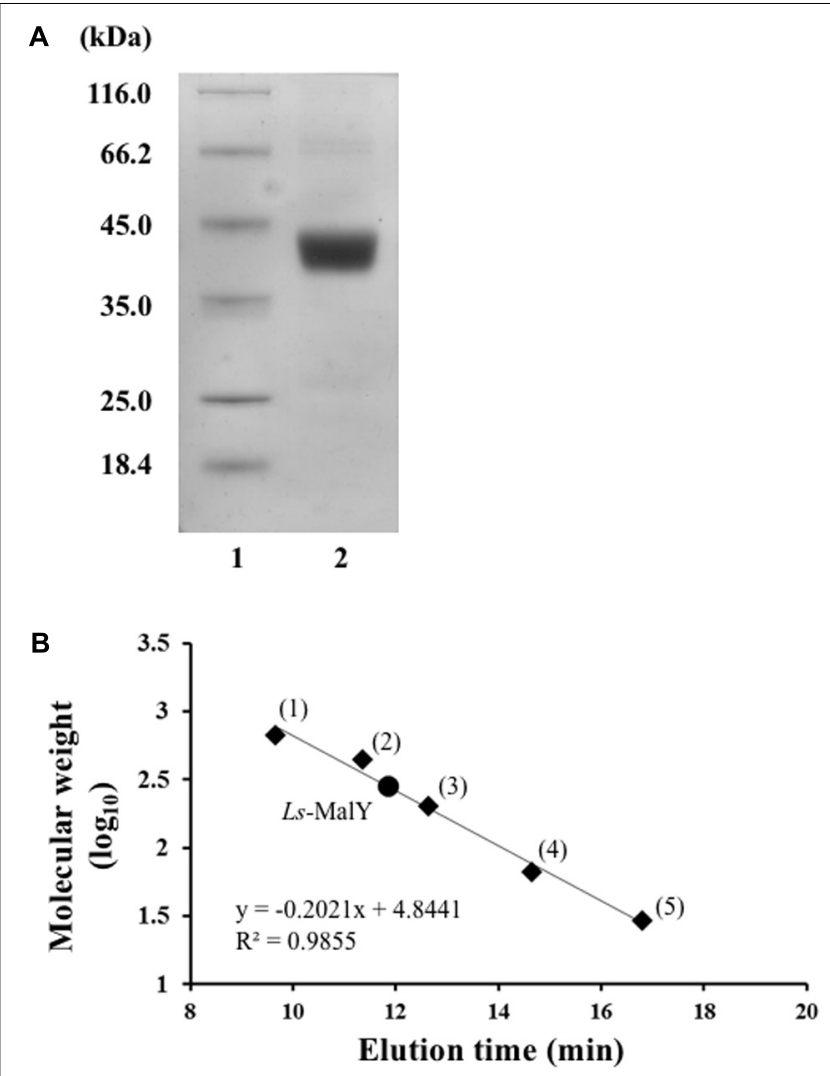

FIGURE 2 | SDS-PAGE (A) and gel filtration chromatographic (B) analyses of WT Ls-MalY. (A) Lanes 1 and 2 indicate molecular weight marker and purified Ls-MalY (4 $\mu \mathrm{g})$, respectively. (B) Thyroglobulin (1: 669,000 Da), apoferritin (2: 443,000 Da), $\beta$-amylase (3: 200,000 Da), BSA (4: 66,000 Da), and carbonic anhydrase (5: 23,000 Da) were used as molecular weight markers.

\section{Purification, Molecular Weight Analysis, and Spectral Measurement of Ls-MalY}

$L s$-MalY overproduced in E. coli was purified to homogeneity using Ni-NTA affinity column chromatography (Figure 2A). From gel filtration column chromatography analysis, the molecular weight of $L s$-MalY was estimated to be approximately $281 \mathrm{kDa}$ (Figure 2B). The molecular weight of the Ls-MalY subunit with a C-terminal hexa-histidine-tag deduced from the amino acid sequence was approximately $46 \mathrm{kDa}$, suggesting that $L s$-MalY is a homohexamer. The UV-vis absorption spectrum of purified $L s$-MalY exhibited an absorption peak near $420 \mathrm{~nm}$, and the absorption peak was abolished by treatment with hydroxylamine (Figure 3A), suggesting that Ls-MalY bound PLP.

\section{Reactivity and Substrate Specificity of Ls-MalY}

High-performance liquid chromatography analysis showed that Ls-MalY can react with L-Ala and D-Ala and catalyze the racemization reaction (Figure 4). The substrate specificity of $L s$-MalY was assessed against proteinogenic amino acids for racemase activity and against Cys and Ser for $\beta$-lyase activity. Ls-MalY exhibited racemase activity with low substrate 

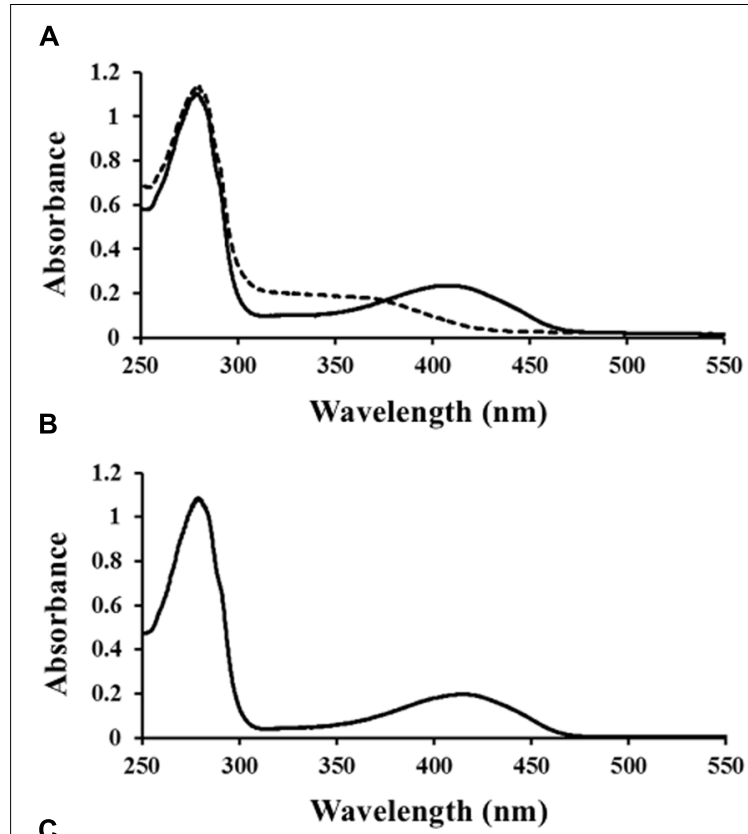

C

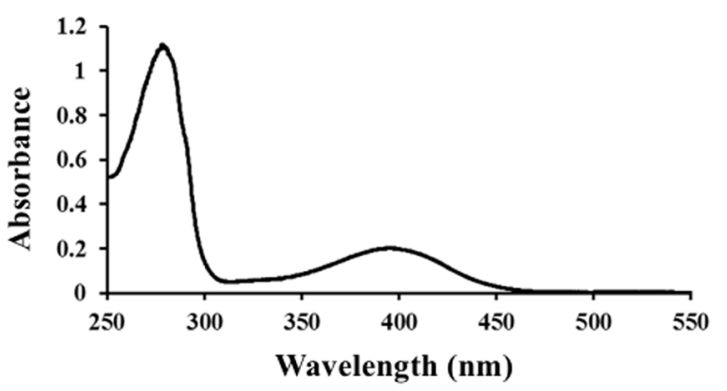

FIGURE 3 | Spectral characteristics of WT (A), Y123A (B), and K233A (C) Ls-MalY. UV-vis spectrum of Ls-MalY. Solid and dashed lines indicate non-treated $L s$-MalY and enzyme treated with $1 \mathrm{mM}$ hydroxylamine in $20 \mathrm{mM}$ potassium phosphate buffer ( $\mathrm{pH} 7.5)$, respectively.

specificity (Table 2) and showed $\beta$-lyase activity toward L-Cys (Table 3). Ls-MalY also showed $\beta$-lyase activity with L-cystine and L-cystathionine (data not shown). However, the specific activity could not be calculated due to the insolubility of the substrates and products. These results indicate that Ls-MalY is a bifunctional amino acid racemase.

\section{Effects of Temperature and $\mathrm{pH}$ on Ls-MalY Activity}

Effects of temperature and $\mathrm{pH}$ on Ls-MalY activity were examined, ranging from 20 to $55^{\circ} \mathrm{C}$ and from $\mathrm{pH} 4.0$ to 12.0 . The optimal temperatures of the racemase and $\beta$-lyase reactions were 45 and $40^{\circ} \mathrm{C}$, respectively (Figures $5 \mathbf{A}, \mathbf{C}$ ). The $\mathrm{pH}$ value optimum was the same for both reactions namely, $\mathrm{pH} 10.0$ (Figures 5B,D).

\section{Kinetic Analysis of Ls-MalY}

The kinetic parameters of $L s$-MalY are listed in Table 4. From these parameters, the $K_{\mathrm{eq}}$ value of the racemization
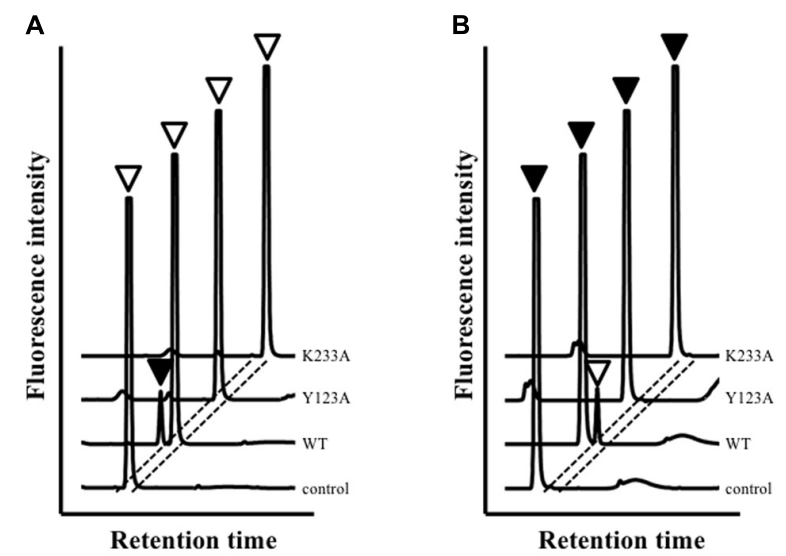

FIGURE 4 | High-performance liquid chromatography (HPLC) analysis of racemase reaction toward L-Ala $(\mathbf{A})$ and D-Ala $(\mathbf{B})$. White and black arrowheads indicate L-Ala and D-Ala peaks, respectively.

TABLE 2 | Substrate specificity of racemase reaction of WT enzyme.

\begin{tabular}{lcc}
\hline Substrate & \multicolumn{2}{c}{ Direction } \\
\cline { 2 - 3 } & $\mathbf{L} \rightarrow \mathbf{D}$ & $\mathbf{D} \rightarrow \mathbf{~}$ \\
\hline Ala & $9.3 \pm 0.3$ & $9.3 \pm 0.5$ \\
Ser & $1.8 \pm 0.1$ & $1.8 \pm 0.1$ \\
Thr & $0.059 \pm 0.002$ & nd \\
Allo-Thr & $0.024 \pm 0.001$ & nd \\
Met & $1.9 \pm 0.1$ & $1.5 \pm 0.1$ \\
Glu & $0.055 \pm 0.007$ & $0.097 \pm 0.064$ \\
Trp & $0.22 \pm 0.01$ & $0.10 \pm 0.03$ \\
Tyr & Trace & Trace \\
Val & $2.5 \pm 0.1$ & $2.4 \pm 0.1$ \\
Leu & $0.13 \pm 0.01$ & $0.096 \pm 0.005$ \\
Asn & $0.71 \pm 0.09$ & $0.44 \pm 0.01$ \\
Gln & $0.57 \pm 0.01$ & $0.57 \pm 0.01$ \\
Lys & $0.92 \pm 0.06$ & $1.0 \pm 0.1$ \\
Arg & $7.6 \pm 0.1$ & $5.8 \pm 0.1$ \\
His & $1.4 \pm 0.1$ & $1.4 \pm 0.1$ \\
\hline The & $\pm v e n$
\end{tabular}

The given numbers are the average of triplicate measurements and shown as specific activity $(\mathrm{nmol} / \mathrm{min} / \mathrm{mg})$. Enzyme assay was performed under standard assay conditions. Other amino acids (Asp, Pro, Phe, lle, and allo-lle) were inert as substrate. nd, not detected.

TABLE 3 | Substrate specificity of $\beta$-lyase reaction.

\begin{tabular}{lccc}
\hline Substrate & WT & Y123A & K233A \\
\hline L-Cys & $95 \pm 1$ & $0.32 \pm 0.11$ & nd \\
D-Cys & nd & nd & nd \\
L-Ser & nd & $0.42 \pm 0.11$ & nd \\
D-Ser & nd & nd & $6.4 \pm 0.1$ \\
\hline
\end{tabular}

The numbers are the average of triplicate measurements and shown as specific activity (nmol/min/mg). Enzyme assays were performed under the standard assay condition. nd, not detected.

reaction between L-Ala and D-Ala was calculated as 1.12, indicating that the enzyme is a racemase (Briggs and Haldane, 1925). The $k_{\text {cat }} / K_{\mathrm{m}}$ values for the racemase 

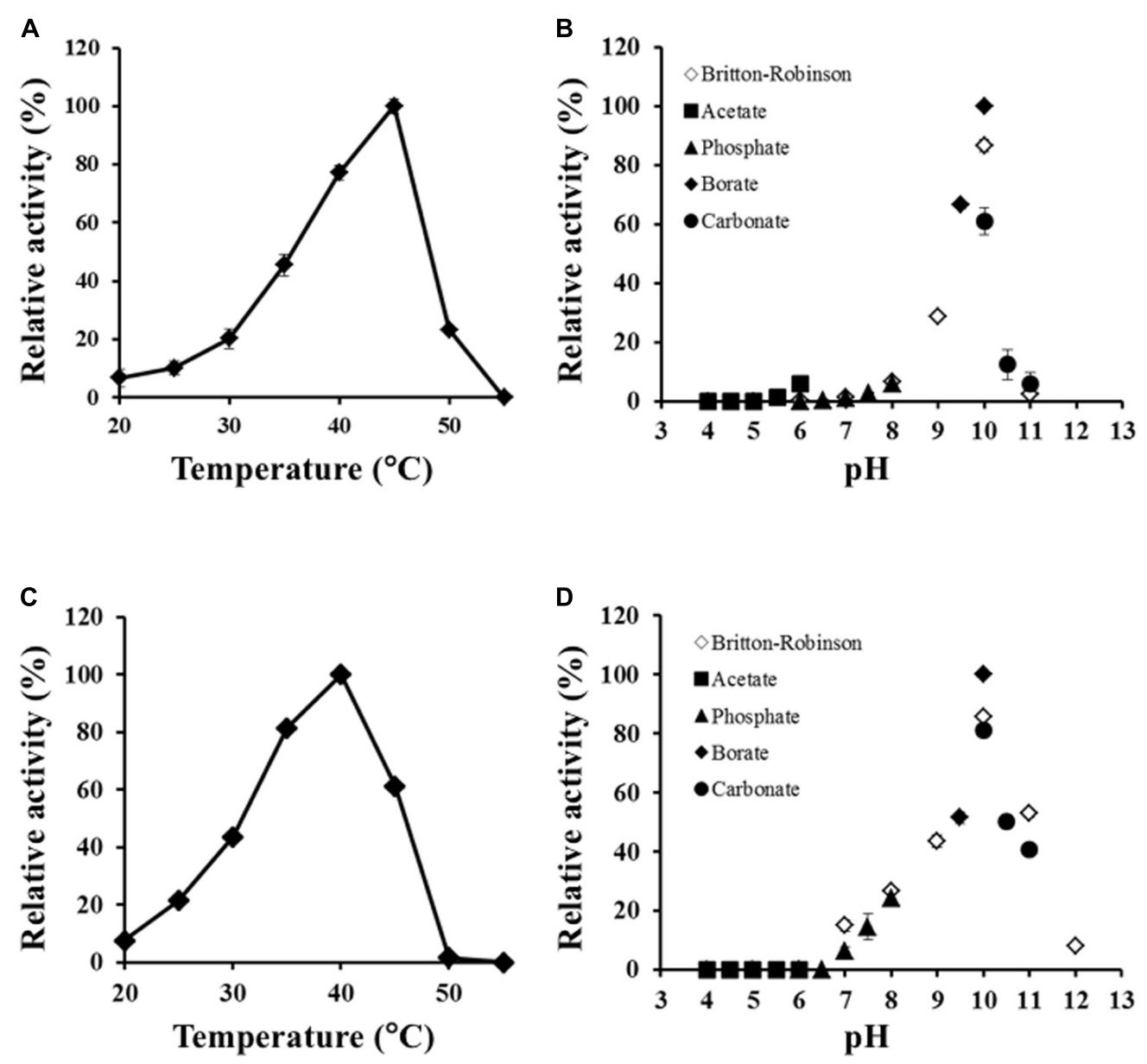

FIGURE 5 | Thermal and $\mathrm{pH}$ profiles of racemase and $\beta$-lyase reactions. Effect of temperature on racemase (A) and $\beta$-lyase $\mathbf{( C )}$ reactions at $\mathrm{pH} 7.5$ (50 $\mathrm{mM}$ potassium phosphate) and $\mathrm{pH}$ dependence of racemase $(\mathbf{B})$ and $\beta$-lyase (D) reactions at $30^{\circ} \mathrm{C}$ were assessed from 20 to $55^{\circ} \mathrm{C}$ and $\mathrm{pH}$ from 4.0 to 12.0 . The indicated values are the average of triplicate measurements.

TABLE 4 | Kinetic parameters of racemase and $\beta$-lyase reactions.

\begin{tabular}{|c|c|c|c|c|}
\hline Reaction & Substrate & $k_{\text {cat }}\left(\mathrm{s}^{-1}\right)$ & $K_{\mathrm{m}}(\mathrm{mM})$ & $k_{\text {cat }} / K_{\mathrm{m}}\left(\mathrm{s}^{-1} \mathrm{mM}^{-1}\right)$ \\
\hline Racemase & L-Ala & $2.31 \pm 0.51 \times 10^{3}$ & $169 \pm 27$ & 13.7 \\
\hline Racemase & D-Ala & $1.83 \pm 0.15 \times 10^{3}$ & $150 \pm 24$ & 12.2 \\
\hline$\beta$-Lyase & L-Cys & $9.30 \pm 2.05 \times 10^{2}$ & $11.5 \pm 1.1$ & 80.9 \\
\hline
\end{tabular}

Enzyme assay was performed at $\mathrm{pH} 10.0$ and $40^{\circ} \mathrm{C}$ (racemase reaction) or $35^{\circ} \mathrm{C}$ ( $\beta$-lyase reaction). Kinetic parameters were calculated with triplicate measurements.

reaction and $\beta$-lyase reaction showed the same order of magnitude. Ls-MalY appears to act as both amino acid racemase and $\beta$-lyase, at least under the enzyme preferred conditions.

\section{Structural Modeling and Identification of Ls-MalY Catalytic Residues}

The amino-acid sequence of Ls-MalY showed high homology with Ec-MalY (identity, 42\%; similarity, 60\%) (Figure 6A). Comparison of the structural model of the Ls-MalY subunit created in this study and Ec-MalY subunit structure (Clausen et al., 2000) suggests that the overall subunit structure and amino acid configuration near the putative catalytic site of $L s$-MalY was quite similar to Ec-MalY (Figures 6B-G). To identify catalytic residues for the racemase and $\beta$-lyase reactions, two single-point mutants (Y123A and K233A) were prepared. K233 of Ls-MalY is a counterpart of PLP-bound K233 of EcMalY and Y123 of Ls-MalY (Y121 of Ec-MalY) is located across the pyridine ring of PLP from K233 (Figures 6E-G). A spectral characteristic of the Y123A mutant (Figure 3B) was quite similar to the WT (Figure 3A), but an absorption peak derived from PLP showed a slight blueshift in the UVvis absorption spectrum of K233A mutant (Figure 3C). These spectral features suggest that the Y123A mutant bound PLP 
and the K233A mutant contained free PLP. Both mutants lost all racemase activity (Figure 4), suggesting that Y123 and K233 are critical residues for the racemase reaction. Y123A mutation also caused a drastic decrease in $\beta$-lyase activity, and the K233A mutant lost $\beta$-lyase activity toward L-Cys (Table 3). K233 is also critical for the $\beta$-lyase reaction with L-Cys, and Y123 appears to be an important residue for the reaction. The $\mathrm{K} 233 \mathrm{~A}$ mutant and Y123A mutant showed $\beta$-lyase activity against $\mathrm{D}$-Ser and L-Ser, respectively. These results suggest that $\mathrm{K} 233$ and $\mathrm{Y} 123$ are responsible for the $\mathrm{C}^{\alpha}$ proton abstraction of L-enantiomers and D-enantiomers, respectively.

\section{Spectral Analysis of Ls-MalY $\beta$-Lyase Reaction}

To solve the reaction mechanism for Ls-MalY, alterations in the UV-vis absorption spectrum at 300-500 nm for WT Ls-MalY during the $\beta$-lyase reaction with L- or D-Cys were examined. An absorption peak at approximately $420 \mathrm{~nm}$ decreased dependent on the dose of substrate and a peak at approximately $330 \mathrm{~nm}$, which might be derived from a reaction intermediate that simultaneously increased when reacted with L-Cys (Figure 7A) or D-Cys (Figure 7B). When reacted with L-Cys (Figure 7C), the spectrum was gradually altered dependent on reaction time, while the spectrum of Ls-MalY reacted with D-Cys was fixed after partial changes (Figure 7D). These results raise the possibility that L-Cys is turned over by Ls-MalY, whereas a deaD-end product may be formed when reacted with D-Cys.

\section{DISCUSSION}

The present study revealed that MalY protein from L. sakei LT-13 is a bifunctional enzyme that can catalyze the amino acid racemase reaction and $\beta$-lyase reaction. The enzyme preferred a moderate temperature for both reactions, in agreement with the L. sakei growth temperature $\left(30-37^{\circ} \mathrm{C}\right)$. The preferred $\mathrm{pH}$ for enzyme activity was in the alkaline range, although the suitable condition for $L$. sakei growth is a weakly acidic environment ( $\mathrm{pH}$ 6.0-6.5). These properties are often found in other PLP-dependent amino acid racemases derived from lactic acid bacteria (Kato et al., 2012; Mutaguchi et al., 2016), and Ls-MalY can display both activities in a weakly acidic condition, suggesting that LsMalY might act as a bifunctional enzyme in L. sakei growing cells.

The MalY protein whose enzyme characteristics are most studied is the protein from E. coli. Ec-MalY has been shown to possess $\beta$-lyase activity (Zdych et al., 1995). The PLP bound K233 of $E c$-MalY is an essential residue for abstraction of the substrate $C^{\alpha}$ proton during the $\beta$-lyase reaction, and the Y121 of $E c$-MalY interacting with pyridine ring of PLP contributes to stabilization of the carbanionic intermediate (Zdych et al., 1995; Clausen et al., 2000). These two residues conserved in Ls-MalY (K233 and Y123) were shown to be important for both the amino acid

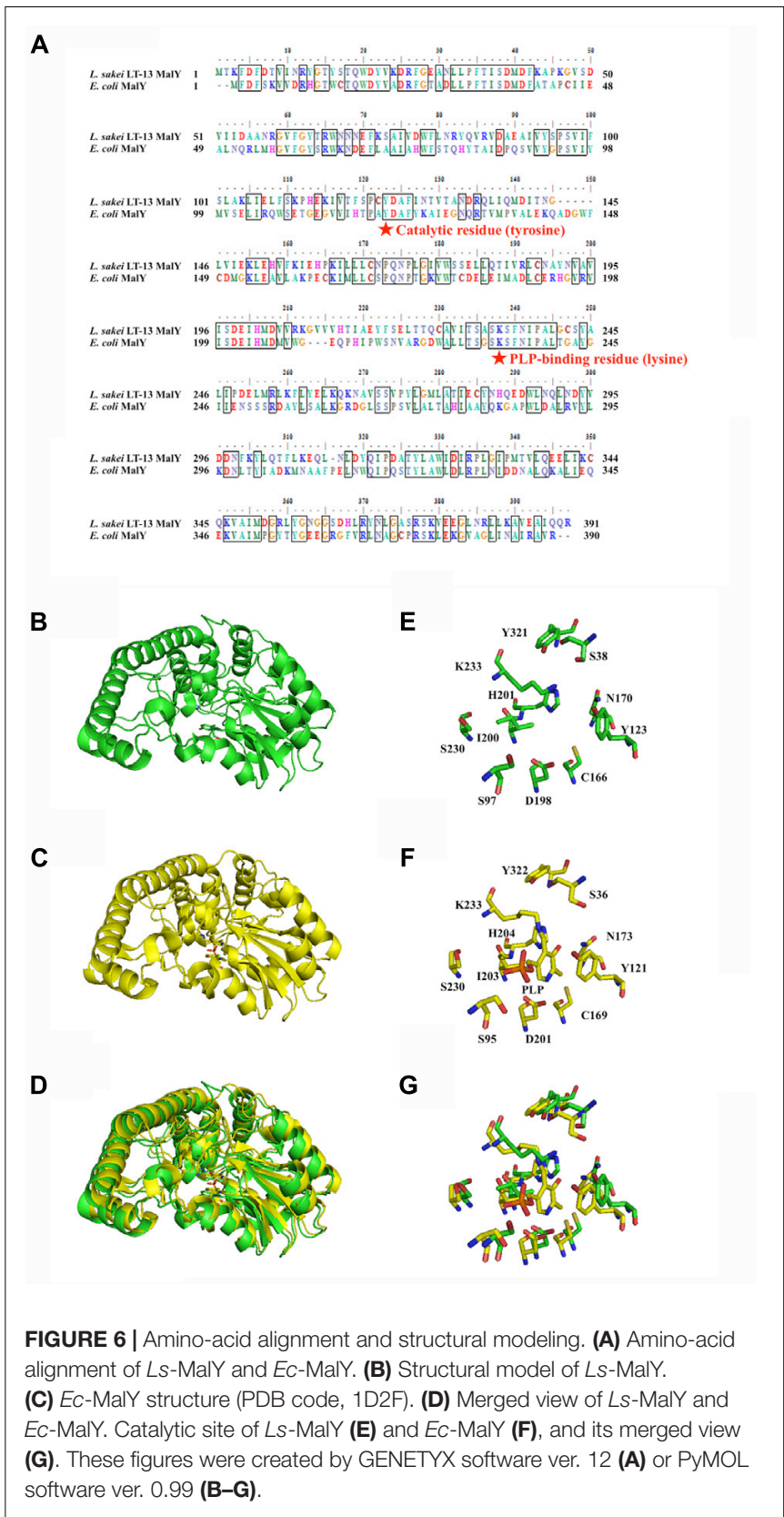

racemase and $\beta$-lyase reactions by mutational analysis, suggesting that both reactions catalyzed by Ls-MalY share common residues for catalysis and that the mechanism of the LsMalY catalyzed $\beta$-lyase reaction is same as for $E c$-MalY. From the configuration of K233 and Y123 in the Ls-MalY structural model and the results of the present mutational analysis, the Ls-MalY catalyzed racemase reaction appears to proceed through a two-base mechanism similar to that of the well-known alanine racemase (Watanabe et al., 2002). Some PLP-dependent enzymes are known to be inhibited by L-Cys and/or D-Cys through the formation of thiazolidine derivatives (Schonbeck et al., 1975; Dunlop and Neidle, 2005; Lowther et al., 2012). The spectral features of Ls-MalY when 

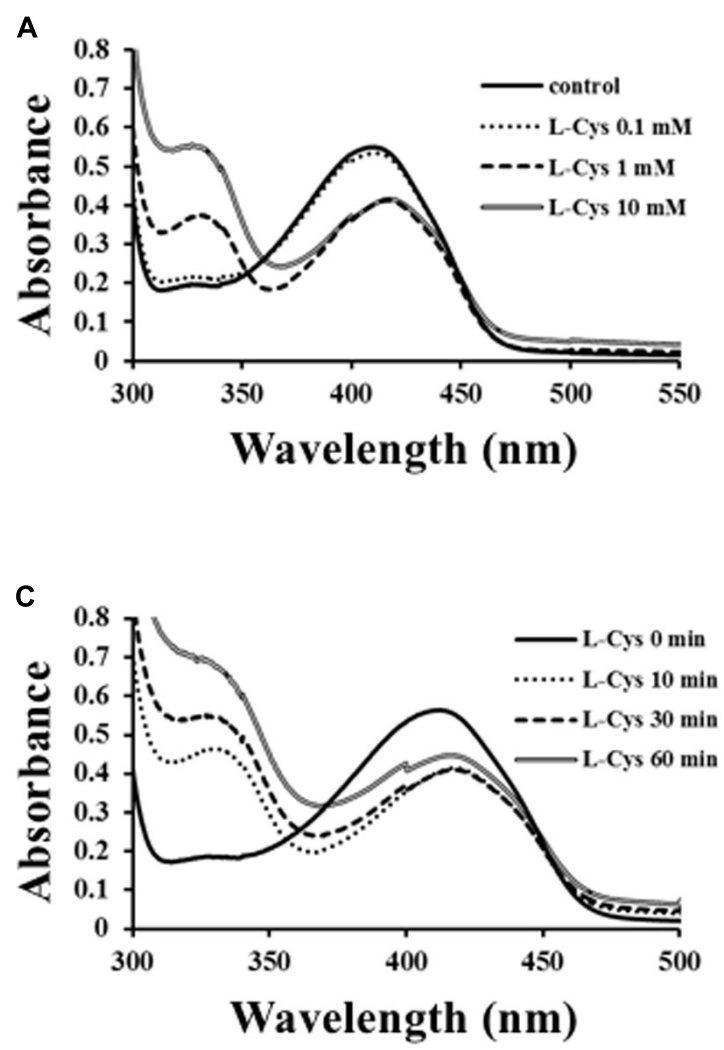
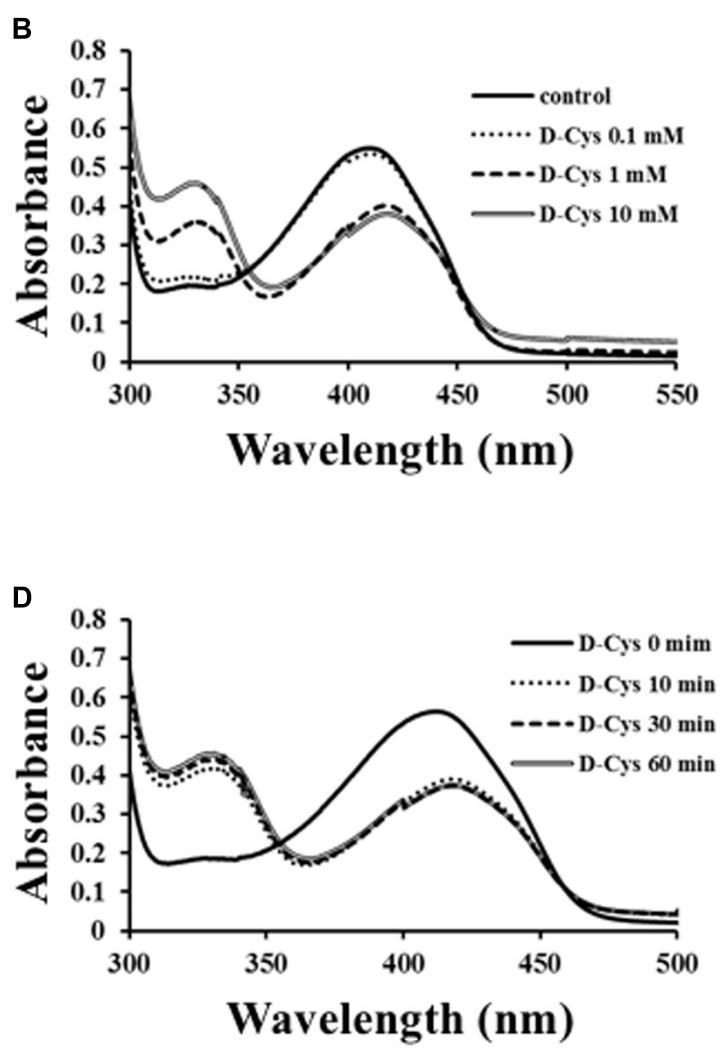

FIGURE 7 | Spectral analysis of $\beta$-lyase reaction. Dose-dependent (A,B) and reaction time-dependent (C,D) changes in UV-vis spectrum of WT Ls-MalY during $\beta$-lyase reaction with L- or D-Cys were assessed from 0 to $10 \mathrm{mM}$ and from 0 to $60 \mathrm{~min}$, respectively.

reacted with D-Cys are in agreement with such reports, suggesting that $L s$-MalY forms a deaD-end product, namely, a thiazolidine adduct, and that $L s$-MalY can catalyze $\beta$-lyase reactions against $\mathrm{L}$-Cys but not racemase reactions between L-Cys and D-Cys. Ec-MalY has also been suggested to be involved in D-amino acid metabolism in E. coli (Kang et al., 2011), but enzyme activity toward D-amino acids has not been reported. To our knowledge, this is the first report that shows amino acid racemase activity for the MalY family protein.

Ls-MalY was identified from the L. sakei strain LT-13, which is a low-level producer of D-amino acids. In contrast, a D-amino acids high producer, the strain LK-145, does not possess MalY protein. Ls-MalY exhibited the highest racemase activity against Ala, and L. sakei LT-13 possesses a putative alanine racemase gene, suggesting that the low D-amino acid producer strain LT13 has two enzymes that can catalyze interconversion between L-Ala and D-Ala. In Salmonella typhimurium, there are also two alanine racemases, Alr and DadB, which have different physiological roles: Alr and DadB are required for anabolic function in peptidoglycan assembly and cell growth on L-Ala, respectively (Walsh, 1989). The two E. coli alanine racemases, Alr and DadX are also involved in various events, including the biosynthesis of D-Ala for peptidoglycan and catabolism of D-Ala (Wild et al., 1985; Lobocka et al., 1994). Ls-MalY may act as DadB or DadX in L. sakei LT-13 cells. The MalY protein is conserved in some species of the Lactobacillus genera, including Lactobacillus casei, and the protein has been shown to possess $\beta$-lyase activity toward some sulfur-containing amino acids; however, reactivity toward D-amino acids has not been reported (Irmler et al., 2008). The relationship between MalY function and D-amino acid metabolism for the Lactobacillus genera is of interest.

The Ls-malY gene (LACBS_00576) is located in a putative $8-\mathrm{kb}$ gene cluster, which is not conserved in strain LK-145. The cluster contains 9 genes (LACBS_00568 to LACBS_00576) encoding proteins expected to be involved in the phosphotransferase system (PTS), based on their primary structure. In E. coli, the malY gene is in malXY operon near its repressor malI gene, and the MalX protein is an enzyme relates to PTS (Reidl and Boos, 1991). Ec-MalY is regarded as a maltose regulon repressor and interacts with MalT protein (Schlegel et al., 2002), which is an essential transcriptional activator of the maltose regulon (Boos and Shuman, 1998). An endogenous ligand for $E c$-MalY that is important for controlling MalT function remains unclear (Clausen et al., 2000), and the reactivity of $L s$-MalY toward D-amino acids presented here raises the possibility that D-amino acids or homologous compounds may be native ligands for the MalY protein. The Ls-malY gene exists in the genome with PTS-related genes similar to the E. coli 
malY gene. However, no candidate gene encoding a homologous protein to MalT from E. coli is observed in the L. sakei LT-13 genome, and genes corresponding to the maltose regulon of E. coli are not fully conserved. We are currently investigating the physiological function(s) of MalY in L. sakei LT-13 cells.

\section{AUTHOR CONTRIBUTIONS}

TO planned this research and organized the entire manuscript. SK did all the practical experiment of this research.

\section{REFERENCES}

Boos, W., and Shuman, H. (1998). Maltose/maltodextrin system of Escherichia coli: transport, metabolism, and regulation. Microbiol. Mol. Biol. Rev. 62, 204-229.

Briggs, G. E., and Haldane, J. B. S. (1925). A note on the kinetics of enzyme action. Biochem. J. 19, 338-339. doi: 10.1042/bj0190338

Cardinale, G. J., and Abeles, R. H. (1968). Purification and mechanism of action of proline racemase. Biochemistry 7, 3970-3978. doi: 10.1021/bi00851a026

Chaillou, S., Champomier-Vergès, M. C., Cornet, M., Crutz-Le Coq, A. M., Dudez, A. M., Martin, V., et al. (2005). The complete genome sequence of the meat-borne lactic acid bacterium Lactobacillus sakei 23K. Nat. Biotechnol. 23, 1527-1533. doi: 10.1038/nbt1160

Choi, S. Y., Esaki, N., Yoshimura, T., and Soda, K. (1992). Reaction mechanism of glutamate racemase, a pyridoxal phosphate-independent amino acid racemase. J. Biochem. 112, 139-142. doi: 10.1093/oxfordjournals.jbchem.a123853

Clausen, T., Schlegel, A., Peist, R., Schneider, E., Steegborn, C., Chang, Y. S., et al. (2000). X-ray structure of MalY from Escherichia coli: a pyridoxal 5(-phosphatedependent enzyme acting as a modulator in mal gene expression. EMBO J. 19, 831-842. doi: 10.1093/emboj/19.5.831

Dunlop, D. S., and Neidle, A. (2005). Regulation of serine racemase activity by amino acids. Brain Res. Mol. Brain Res. 133, 208-214. doi: 10.1016/j. molbrainres.2004.10.027

Fujii, T., Yamauchi, T., Ishiyama, M., Gogami, Y., Oikawa, T., and Hata, Y. (2015). Crystallographic studies of aspartate racemase from Lactobacillus sakei NBRC 15893. Acta Crystallogr. F Struct. Biol. Commun. 71, 1012-1016. doi: 10.1107/ S2053230X15010572

Gogami, Y., Okada, K., and Oikawa, T. (2011). High-performance liquid chromatography analysis of naturally occurring D-amino acids in sake. J. Chromatogr. B 879, 3259-3267. doi: 10.1016/j.jchromb.2011.04.006

Grabski, A., Mehler, M., and Drott, D. (2005). The overnight express autoinduction system: high-density cell growth and protein expression while you sleep. Nat. Methods 2, 233-235. doi: 10.1038/nmeth0305-233

Irmler, S., Raboud, S., Beisert, B., Rauhut, D., and Berthoud, H. (2008). Cloning and characterization of two Lactobacillus casei genes encoding a cystathionine lyase. Appl. Environ. Microbiol. 74, 99-106. doi: 10.1128/AEM.00745-07

Kang, L., Shaw, A. C., Xu, D., Xia, W., Zhang, J., Deng, J., et al. (2011). Upregulation of MetC is essential for D-alanine-independent growth of an alr/dadX-deficient Escherichia coli strain. J. Bacteriol. 193, 1098-1106. doi: 10.1128/JB.01027-10

Kato, S., Hemmi, H., and Yoshimura, T. (2012). Lysine racemase from a lactic acid bacterium, Oenococcus oeni: structural basis of substrate specificity. J. Biochem. 152, 505-508. doi: 10.1093/jb/mvs 120

Kato, S., Ishihara, T., Hemmi, H., Kobayashi, H., and Yoshimura, T. (2011). Alterations in D-amino acid concentrations and microbial community structures during the fermentation of red and white wines. J. Biosci. Bioeng. 111, 104-108. doi: 10.1016/j.jbiosc.2010.08.019

Kato, S., Masuda, Y., Konishi, M., and Oikawa, T. (2015). Enantioselective analysis of $\mathrm{D}$-and L-amino acids from mouse macrophages using high performance liquid chromatography. J. Pharm. Biomed. Anal. 116, 101-104. doi: 10.1016/j. jpba.2015.04.028

Kato, S., and Oikawa, T. (2017a). Genome sequence of Lactobacillus sakei LK-145 isolated from a Japanese sake cellar as a high amount of D-amino acid producer. Genome Announc. 5:e00656-17. doi: 10.1128/genomeA.00656-17

\section{FUNDING}

This work was supported by the Ministry of Education, Culture, Sports, Science and Technology (MEXT)-Supported Program for the Strategic Research Foundation at Private Universities, 2013-2017.

\section{ACKNOWLEDGMENTS}

The authors thank Kiku-Masamune Sake Brewing, Co., Ltd. for their kind gift of Lactobacillus sakei strains.

Kato, S., and Oikawa, T. (2017b). Whole genome sequence of Lactobacillus sakei LT-13 isolated from moto starter of sake. Genome Announc. 5:e651-17. doi: 10.1128/genomeA.00651-17

Kolodkin-Gal, I., Romero, D., Cao, S., Clardy, J., Kolter, R., and Losick, R. (2010). D-Amino acids trigger biofilm disassembly. Science 328, 627-629. doi: 10.1126/ science. 1188628

Lam, H., Oh, D. C., Cava, F., Takacs, C. N., Clardy, J., de Pedro, M. A., et al. (2009). $\mathrm{D}$-Amino acids govern stationary phase cell wall remodeling in bacteria. Science 325, 1552-1555. doi: 10.1126/science. 1178123

Li, C., and Lu, C. D. (2009). Arginine racemization by coupled catabolic and anabolic dehydrogenases. Proc. Natl. Acad. Sci. U.S.A. 106, 906-911. doi: $10.1073 /$ pnas.0808269106

Lineweaver, H., and Burk, D. (1934). The determination of enzyme dissociation constants. J. Am. Chem. Soc. 56, 658-666. doi: 10.1021/ja01318a036

Lobocka, M., Hennig, J., Wild, J., and Kłopotowski, T. (1994). Organization and expression of the Escherichia coli K-12 dad operon encoding the smaller subunit of D-amino acid dehydrogenase and the catabolic alanine racemase. J. Bacteriol. 176, 1500-1510. doi: 10.1128/jb.176.5.1500-1510.1994

Lowther, J., Beattie, A. E., Langridge-Smith, P. R., Clarke, D. J., and Campopiano, D. J. (2012). L-Penicillamine is a mechanism-based inhibitor of serine palmitoyltransferase by forming a pyridoxal-5'-phosphate-thiazolidine adduct. Med. Chem. Commun. 3, 1003-1008. doi: 10.1039/c2md2 0020a

Matsui, D., Oikawa, T., Arakawa, N., Osumi, S., Lausberg, F., Stäbler, N., et al. (2009). A periplasmic, pyridoxal-5'-phosphate-dependent amino acid racemase in Pseudomonas taetrolens. Appl. Microbiol. Biotechnol. 83, 1045-1054. doi: 10.1007/s00253-009-1942-7

Miyamoto, T., Katane, M., Saitoh, Y., Sekine, M., and Homma, H. (2017). Identification and characterization of novel broad-spectrum amino acid racemases from Escherichia coli and Bacillus subtilis. Amino Acids 49, 1885-1894. doi: 10.1007/s00726-017-2486-2

Moriya, Y., Itoh, M., Okuda, S., Yoshizawa, A. C., and Kanehisa, M. (2007). KAAS: an automatic genome annotation and pathway reconstruction server. Nucleic Acids Res. 35(Suppl._2), W182-W185. doi: 10.1093/nar/gkm321

Mutaguchi, Y., Kobayashi, Y., Oikawa, T., and Ohshima, T. (2016). "D-amino acids in fermentative foods," in D-Amino Acids Physiology Metabolism and Application, eds T. Yoshimura, T. Nishikawa, and H. Homma (New York, NY: Springer), 341-357.

Mutaguchi, Y., Ohmori, T., Akano, H., Doi, K., and Ohshima, T. (2013a). Distribution of $\mathrm{D}$-amino acids in vinegars and involvement of lactic acid bacteria in the production of D-amino acids. Springerplus 2, 691-699. doi: 10.1186/2193-1801-2-691

Mutaguchi, Y., Ohmori, T., Wakamatsu, T., Doi, K., and Ohshima, T. (2013b). Identification, purification, and characterization of a novel amino acid racemase, isoleucine 2-epimerase, from Lactobacillus species. J. Bacteriol. 195, 5207-5215. doi: 10.1128/JB.00709-13

Oikawa, T., Tauch, A., Schaffer, S., and Fujioka, T. (2006). Expression of alr gene from Corynebacterium glutamicum ATCC 13032 in Escherichia coli and molecular characterization of the recombinant alanine racemase. J. Biotechnol. 125, 503-512. doi: 10.1016/j.jbiotec.2006.04.002

Okada, K., Gogami, Y., and Oikawa, T. (2013). Principal component analysis of the relationship between the D-amino acid concentrations and the 
taste of the sake. Amino Acids 44, 489-498. doi: 10.1007/s00726-0121359-y

Reidl, J., and Boos, W. (1991). The malX malY operon of Escherichia coli encodes a novel enzyme II of the phosphotransferase system recognizing glucose and maltose and an enzyme abolishing the endogenous induction of the maltose system. J. Bacteriol. 173, 4862-4876. doi: 10.1128/jb.173.15.4862-4876. 1991

Schlegel, A., Danot, O., Richet, E., Ferenci, T., and Boos, W. (2002). The N terminus of the Escherichia coli transcription activator MalT is the domain of interaction with MalY. J. Bacteriol. 184, 3069-3077. doi: 10.1128/JB.184.11.3069-3077. 2002

Schonbeck, N. D., Skaiski, M., and Shafer, J. A. (1975). Resolution of D-serine dehydratase by cysteine. An analytical treatment. J. Biol. Chem. 250, 5352-5358.

Soda, K. (1968). Microdetermination of D-amino acids and D-amino acid oxidase activity with 3-methyl-2-benzothiazolone hydrazone hydrochloride. Anal. Biochem. 25, 228-235. doi: 10.1016/0003-2697(68)90095-X

Studier, F. W. (2005). Protein production by auto-induction in high-density shaking cultures. Protein Expr. Purif. 41, 207-234. doi: 10.1016/j.pep.2005. 01.016

Walsh, C. T. (1989). Enzymes in the D-alanine branch of bacterial cell wall peptidoglycan assembly. J. Biol. Chem. 264, 2393-2396.

Washio, T., Kato, S., and Oikawa, T. (2016). Molecular cloning and enzymological characterization of pyridoxal 5(-phosphate independent aspartate racemase from hyperthermophilic archaeon Thermococcus litoralis DSM 5473. Extremophiles 20, 711-721. doi: 10.1007/s00792-016-0860-8
Watanabe, A., Yoshimura, T., Mikami, B., Hayashi, H., Kagamiyama, H., and Esaki, N. (2002). Reaction mechanism of alanine racemase from Bacillus stearothermophilus: $\mathrm{x}$-ray crystallographic studies of the enzyme bound with N-(50-phosphopyridoxyl)alanine. J. Biol. Chem. 277, 19166-19172. doi: 10. 1074/jbc.M201615200

Wild, J., Hennig, J., Lobocka, M., Walczak, W., and Klopotowski, T. (1985). Identification of the dadX gene coding for the predominant isozyme of alanine racemase in Escherichia coli K12. Mol. Gen. Genet. 198, 315-322. doi: 10.1007/ BF00383013

Yoshimura, T., Ashiuchi, M., Esaki, N., Kobatake, C., Choi, S. Y., and Soda, K. (1993). Expression of glr (murI, dga) gene encoding glutamate racemase in Escherichia coli. J. Biol. Chem. 268, 24242-24246.

Zdych, E., Peist, R., Reidl, J., and Boos, W. (1995). MalY of Escherichia coli is an enzyme with the activity of a beta C-S lyase (cystathionase). J. Bacteriol. 177, 5035-5039. doi: 10.1128/jb.177.17.5035-5039.1995

Conflict of Interest Statement: The authors declare that the research was conducted in the absence of any commercial or financial relationships that could be construed as a potential conflict of interest.

Copyright (c) 2018 Kato and Oikawa. This is an open-access article distributed under the terms of the Creative Commons Attribution License (CC BY). The use, distribution or reproduction in other forums is permitted, provided the original author(s) and the copyright owner are credited and that the original publication in this journal is cited, in accordance with accepted academic practice. No use, distribution or reproduction is permitted which does not comply with these terms. 